\title{
CRÍTICA AL CRECIMIENTO ECONÓMICO
}

\author{
FERNANDA JARAMILLO \\ Universidad Central del Ecuador
}

Recepción manuscrito: 1 de julio de 2014

Aceptación versión final: 30 de octubre de 2014

\begin{abstract}
RESUMEN El presente artículo realiza una crítica al crecimiento económico ilimitado como medio para alcanzar el desarrollo en una economía y como herramienta para medir el bienestar de la sociedad. En la primera sección se analiza cómo surge esta idea desde la teoría económica neoclásica, para posteriormente, en la siguiente sección, presentar las limitaciones y discrepancias que surgen al creer que el crecimiento económico es un sinónimo de crecimiento y bienestar. Principalmente el artículo se centra en la dimensión ecológica por ser el principal y más obvio limitante al crecimiento. Para finalizar se presenta uno de los planteamientos alternativos más recientes, el decrecimiento económico.
\end{abstract}

palabras ClaVe Crecimiento, bienestar, límites, economía ecológica

ABSTRACT This article takes a critique of unlimited economic growth as a means to achieve development in an economy and as a tool to measure well-being of society. The first section examines how this idea comes from neoclassical economic theory, later, in the next section, present limitations and discrepancies that arise in believing that economic growth is synonymous with growth and welfare. Mainly article focuses on the ecological dimension as the main and most obvious constraint to growth. Finally comes one of the most recent alternative approaches, economic decline.

KEY WORDS Growth, welfare, limits, ecological economics

JEL CODE I31, O41, O47, Q57

\section{INTRODUCCIÓN}

Nos resulta común escuchar aún en la actualidad a los analistas decir que un país se encuentra en una mala situación económica y social cuando sus más conocidos indicadores macroeconómicos, como el Producto Interno Bruto (PIB), presentan cifras bajas o negativas, o por el contrario decir que un país ha aplicado una correcta política y se encuentra en desarrollo debido a que presenta un alto crecimiento económico. Por otra parte, la discusión sobre cuál es la manera más acertada de medir el bienestar socioeconómico es antigua, y ha determinado que existe una amplia cantidad de aspectos que no son considerados por estos indicadores, y que a su vez 
son elementos esenciales para el estudio de la situación del bienestar humano. ¿Es acaso el crecimiento económico la respuesta para alcanzar el desarrollo y bienestar social que todos anhelamos? ¿Es posible que todos los países crezcan económicamente y que éste sea el medio apropiado para mejorar su situación social? Muchos conocemos la respuesta: No. Sin embargo, los esfuerzos de los países siguen esa misma dirección y parecen tener un único fin, el crecimiento económico ilimitado.

El presente artículo analizará dónde y cómo nace la idea de que el crecimiento económico es una medida de desarrollo y bienestar social. Posteriormente se presentan las limitaciones de este planteamiento, principalmente aquellas relacionadas con el hecho de que vivimos en un mundo con recursos naturales finitos que no permiten que el modelo económico actual sea sostenible en el tiempo; y finalmente concluir con uno de los recientes planteamientos alternativos: El llamado decrecimiento económico.

\section{EL CRECIMIENTO ECONÓMICO DESDE EL PARADIGMA NEOCLÁSICO}

El análisis de la economía desde la escuela neoclásica se basa en los supuestos de que el mercado se desarrolla en competencia perfecta, donde los agentes económicos cuentan con total información, existe gran cantidad de compradores y vendedores, no existen barreras al ingreso o salida de competidores, etc. (Miller, 2002, p. 561); y asume que los agentes económicos se manejan bajo un comportamiento racional (homo æeconomicus) caracterizado por la búsqueda egoísta del interés individual, la misma que aportaría para alcanzar el bienestar de toda la sociedad.

Dentro de esta misma escuela, la economía del bienestar considera en primer lugar la maximización de la eficiencia en el punto donde todo equilibrio competitivo cumple con optimalidad de Pareto, es decir que no existe otra distribución por medio de la cual se genere un incremento en la utilidad de un individuo sin disminuir la utilidad de otro; y en segundo lugar se considera la política distributiva para alcanzar la equidad (Falconí, 2002, p. 28).

Además de estos supuestos, la economía neoclásica basa sus teorías en parámetros no físicos como tecnologías, preferencias, distribución del ingreso, etc. Estos parámetros llegan a ser las variables determinantes del equilibrio (o de una tasa de crecimiento de equilibrio). Mientras que los parámetros físicos como los recursos naturales y los bienes producidos, constituyen variables que se ajustan para encajar en el equilibrio fijado. En la teoría neoclásica este 'ajuste' casi siempre implica crecimiento (Daly, 1996, p. 4).

Tradicionalmente la economía ha sido concebida como un sistema circular cerrado, en donde, se asume que los intercambios entre agentes económicos son alimentados a través del dinero de forma perpetua, sin dar cabida alguna a la existencia de un rol explícito de la naturaleza, ni a la consideración de límites a tales intercambios (Kerschner, 2009, p. 6).

La corriente neoclásica concibe a la economía como un sistema circular cerrado que encierra a empresas y familias como actores que intervienen en el mercado de bienes y servicios y en el mercado de factores de producción, a través de flujos monetarios y de bienes y servicios.

Sin embargo, este sistema no considera que la economía extrae mensualmente enormes cantidades de recursos naturales como minerales, petróleo, madera, agua, entre muchos otros, con la finalidad de utilizarlos directamente o integrarlos y transformarlos mediante procesos 
Figura 1. La economía como sistema cerrado según a la economía neoclásica

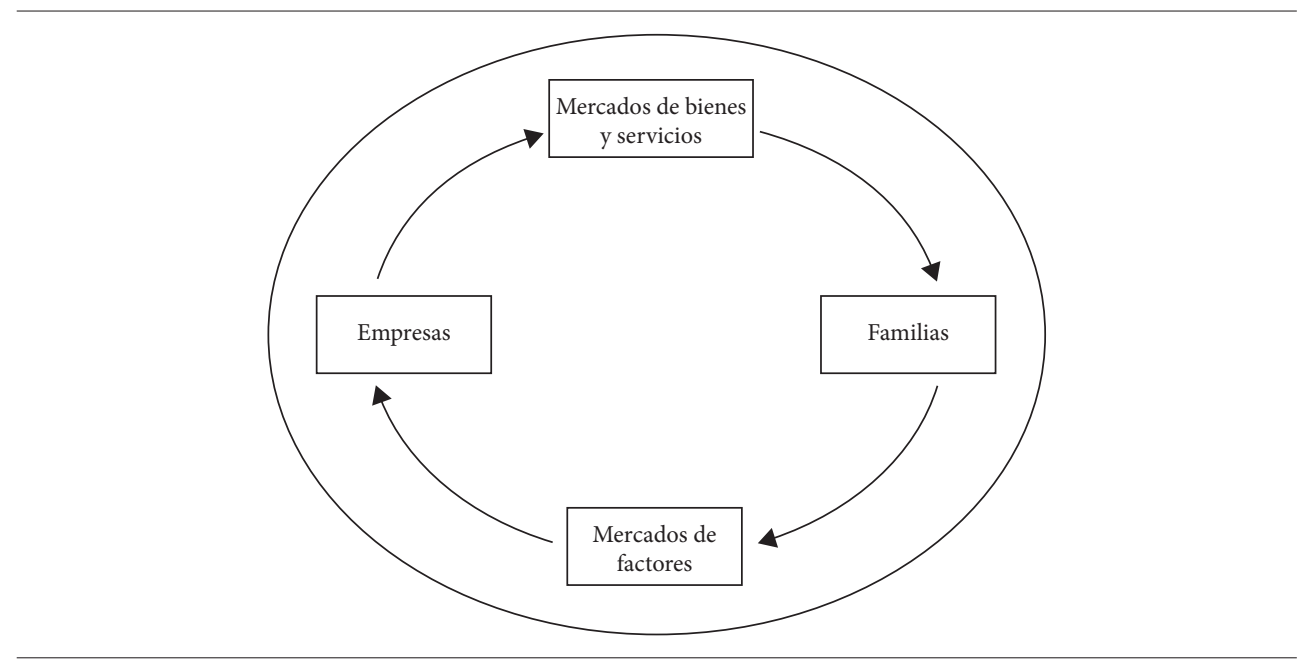

Fuente: adoptado de Martínez-Alier y Roca (2006).

productivos en millones de productos de consumo para los seres humanos. Proceso tras el cual se generan residuos que son devueltos al ambiente.

Posteriormente, a partir de la segunda mitad del siglo XVIII, con el surgimiento de la Revolución Industrial, tomó fuerza la idea de un crecimiento económico constante e ilimitado. En época de la Gran Depresión (1929) y a partir de la publicación en 1936 de la obra Teoría general del empleo, el interés y el dinero (Keynes, 1936), surge el pensamiento keynesiano, el cual considera indispensable la intervención estatal para estimular la demanda agregada a través del incremento del gasto público, asumiéndose así la búsqueda del crecimiento del consumo sin considerar límite alguno.

\section{EL CRECIMIENTO ECONÓMICO COMO MEDIDA DE DESARROLLO Y BIENESTAR}

La búsqueda del desarrollo y el bienestar social ha constituido uno de los principales puntos de estudio en las ciencias sociales. Dentro del análisis de la economía, el pensamiento predominante a lo largo de la historia ha sido que la prosperidad económica, representada por el continuo crecimiento, es el único medio para alcanzarlo. La economía neoclásica considera al crecimiento económico como una necesidad:

Los economistas ortodoxos están convencidos de que sólo el crecimiento económico puede resolver los «problemas clásicos» de la sociedad, formulados por Smith (la pobreza), Malthus (superpoblación), Marx (la distribución) y Keynes (desempleo involuntario). No sólo eso, también es [planteado] como uno (o el único) modo de solucionar los problemas de contaminación, el [pago] de la deuda [externa], [el equilibrio en] la balanza de pagos, el agotamiento de los recursos naturales, la delincuencia, etc. (Kerschner, 2009, p. 8). 
Es decir, tradicionalmente se comete el error de pensar que el crecimiento es la solución para muchos de los problemas económicos: para combatir la pobreza basta incrementar la producción y estimular el gasto; para enfrentar al desempleo se deben disminuir las tasas de interés de préstamos y estimular la inversión para provocar un aumento en la demanda de bienes y servicios, que a su vez genere empleo; la sobrepoblación se controla y remedia al llegar a altos niveles de renta; los daños ambientales disminuirán cuando se llegue al punto de inflexión descrito por la curva ambiental de Kuznets; la redistribución de la renta no debe ser aplicada en función de que ralentiza el proceso de crecimiento económico (Daly, 2005, p. 100).

Bajo este pensamiento los esfuerzos se enfocaron en generar acumulación de capital, dejando sin atención alguna a las consecuencias que esto acarrearía. Surgen entonces profundos problemas sociales y ambientales que con el pasar de los años se hacen más evidentes, y emergen con fuerza interrogantes que buscan definir la relación real entre crecimiento económico y bienestar.

Esta discusión no es reciente, a partir de la década de los años setenta se genera el cambio de posturas, los analistas llegan a darse cuenta de que un país con altos niveles de crecimiento económico reflejado en el PIB, no necesariamente alcanzaban una mejora en la calidad de vida de sus habitantes y no lograban condiciones para un desarrollo integral (Schuldt, 1995, p. 32). Entonces, la visión de prosperidad basada en la expansión económica fue desechada. Algunos estudios revelan que otros factores son esenciales dentro de este análisis, como por ejemplo las relaciones sociales, la situación espiritual, la salud, entre otros; es decir, el crecimiento económico dejó de ser considerado como el elemento trascendental e indispensable para alcanzar el bienestar social (Jackson, 2011, p. 35-49).

De forma similar, en respuesta a la creciente evidencia de una problemática ambiental, a partir del año 1987 surge dentro del análisis económico el concepto de desarrollo sostenible, el mismo que buscaba empatar el crecimiento económico con la problemática ambiental (Munda, 1997, p. 214).

Esta corriente sostiene que la expansión económica es una condición indispensable para alcanzar una prosperidad duradera a largo plazo. Existen tres elementos en defensa de esta afirmación. En primer lugar, la opulencia (como satisfacción material) es indispensable para progresar y desarrollarse en numerosos ámbitos. Así, una mayor cantidad de ingresos implicaría mejores oportunidades, mayor calidad de vida, mayor nivel de acceso a servicios y beneficios, vidas más ricas que permitan elevar el bienestar de las personas. Segundo, el crecimiento económico está altamente correlacionado con nociones esenciales como la salud y la educación; y tercero es que el crecimiento mantiene una estabilidad económica y social. No obstante, si bien el crecimiento económico es considerado por numerosos economistas como una condición necesaria para alcanzar el bienestar, es indispensable contemplar que existen límites tanto físicos como naturales que lo hacen insostenible (Jackson, 2001, p. 49-65).

Los defensores de la expansión económica sostienen que la eficiencia tecnológica se ha convertido en el elemento que permite que el modelo de crecimiento sea viable y compatible con los objetivos ecológicos, e incluso afirman que es necesaria para alcanzarlos. Sin embargo, la evidencia a través de la historia demuestra que esta afirmación es errada; a pesar del acelerado desarrollo tecnológico de las últimas décadas, el consumo y pérdida de recursos 
continúa en aumento, al igual que los daños ambientales que continúan en el camino de tornarse irreversibles (Jackson, 2011, p. 121).

\section{LÍMITES DEL PARADIGMA TRADICIONAL}

La economía, y en general la concepción tradicional de las relaciones entre la sociedad y la naturaleza, han sido mal concebidas desde sus inicios y en relación a varias materias. El desarrollo de todos los aspectos de la vida común en nuestro tiempo, se sustenta en esquemas donde el actor prevaleciente e importante es el Hombre. Es importante destacar la gran capacidad que han tenido los seres humanos para lograr alterar drásticamente y en un tiempo relativamente corto al sistema en el que vivimos. Los seres humanos estamos generando daños irreversibles al planeta Tierra, a la naturaleza que ha vivido en armonía durante millones de años, y a todo este sistema en general.

Pero el origen del problema no emerge en las últimas décadas donde la crisis ya se ha evidenciado; el problema nace mucho más atrás en el tiempo. Los inicios de este asunto surgen a partir las épocas más remotas, desde que en la Biblia se estableció que: «[...] Dios los bendijo [al hombre y a la mujer] diciendo: Creced y multiplicaos, llenad la tierra y dominadla» (Gn 1, 28, citado en Max-Neef, 1986, p. 21), sentando las bases y dando forma así a nuestra cultura. Esto es calificado por Max-Neef como el «mito original» que ha definido el curso de la historia (Max-Neef, 1986, p. 21, 45).

Se deja por sentado entonces que los humanos son los seres superiores que deben crecer sin respeto o límite alguno para dominar el sistema, siendo la naturaleza un recurso que se ponía a plena disposición del Hombre para su completo desarrollo. Surge entonces una civilización irresponsable, que ha generado daños irreversibles en el sistema natural, y ha llevado al límite a sus capacidades.

Las principales ideologías que han surgido en la historia, como el liberalismo, el socialismo, etc.; concuerdan en que el crecimiento económico es un elemento indispensable para la economía; sobreestiman el papel de la tecnología como solución a los problemas, e ignoran el poder e importancia de la naturaleza como elemento esencial para el sustento de la humanidad (Max-Neef, 1986, p. 45).

Los modos de vida adoptados por los seres humanos se vuelven cada vez más agresivos contra la naturaleza, y no se llega a concebir siquiera la posibilidad de un proceso de cambio de vida y de cultura. Es lo que nos ha llevado a vivir una crisis civilizatoria, que para muchos es inexistente.

A pesar de todo, existen nuevas corrientes como la economía ecológica, o propuestas como el decrecimiento económico, que buscan afrontar los problemas de los cuales somos responsables, y realizan un análisis más amplio considerando aquellos elementos que la teoría tradicional no consideró.

Como ya se mencionó, una de las propuestas alternativas que hace frente al crecimiento económico ilimitado es la economía ecológica, la cual incluye dentro del análisis a los flujos de materiales y energía generados durante los procesos físicos, sociales y económicos. Se plantea entonces como un sistema abierto que trasciende el esquema cerrado planteado por la economía neoclásica. 
Este sistema recibe ingresos de energía y materiales, y a su vez emite energía degradada y residuos materiales; parte de estos últimos pueden ser reincorporados como materias primas para la producción, tras pasar por un proceso de reciclaje. La economía como tal es un sistema abierto al ingreso de la energía solar, tal como lo expresaron Martínez-Alier y Roca (ver Figura 2).

\section{LOS LÍMITES DEL CRECIMIENTO}

Bajo el enfoque abierto de la economía ecológica, el crecimiento económico sin límites es incompatible y se presenta como un dilema. Si bien se reconoce que un país pobre requiere de crecimiento económico para que sus habitantes alcancen una buena calidad de vida; también cabe reconocer que aquellas economías con altos niveles de ingresos que ya han logrado cubrir las principales necesidades de sus habitantes, destinan una amplia proporción del ingreso en consumos que pueden considerarse como innecesarios y suntuosos.

Lo seres humanos se han formado en un sistema y sociedad que presenta a la elevada capacidad de consumo y al lujo como el equivalente del desarrollo y éxito, tanto personal como social. Las personas tienen apetitos insaciables y se crean cada vez nuevas necesidades que llevan evidentemente a continuar elevando el consumo. Antropólogos e investigadores de esta temática indican que:

Los bienes de consumo proporcionan un lenguaje simbólico mediante el que nos comunicamos con los demás, no sólo en un sentido estrictamente material, sino sobre lo que realmente nos importa: la familia, la amistad, el sentido de pertenencia, la comunidad, la identidad, el estatus social, el significado y el propósito de la existencia (Jackson, 2011, p. 76-77).

El ingreso, destinado al consumo, es considerado entonces como un factor indispensable para el bienestar de las personas; el estatus en las sociedades se encuentra directamente relacionado con los niveles de ingresos, que a su vez va acompañado de clase social, autoridad, poder, etc. No obstante, el apetito y los esquemas actuales no son compatibles con la limitada capacidad ambiental.

Los límites naturales al crecimiento son abordados con fuerza desde el año 1970, en que la asociación privada conocida como el «Club de Roma», encargó al Massachusetts Institute of Technology (MIT) realizar una investigación que sería publicada en el año 1972, y que marcaría el inicio del interés y preocupación en la real dimensión de la escasez de los recursos naturales. El informe se denominó «Los límites del crecimiento». Los resultados mostraban que:

La Humanidad no puede proliferarse a una tasa acelerada y considerar el desarrollo material como su principal objetivo, sin encontrar obstáculos a este proceso; [...] estamos ante la alternativa de buscar nuevos objetivos para tomar nuestro destino en nuestras propias manos o someternos a las consecuencias inevitablemente más crueles del crecimiento irrestricto. (Meadows et al., 1972, p. 21-22)

Junto al nacimiento de una verdadera preocupación por los límites de la capacidad ambiental, se inicia el debate entre dos enfoques: los denominados optimistas y los pesimistas. Los optimistas, en su mayor parte economistas neoclásicos, defendían con fuerza la posición de que los límites al crecimiento desde el punto de vista ambiental no constituían problema alguno 
Figura 2. La economía como sistema abierto de acuerdo a la economía ecologíca

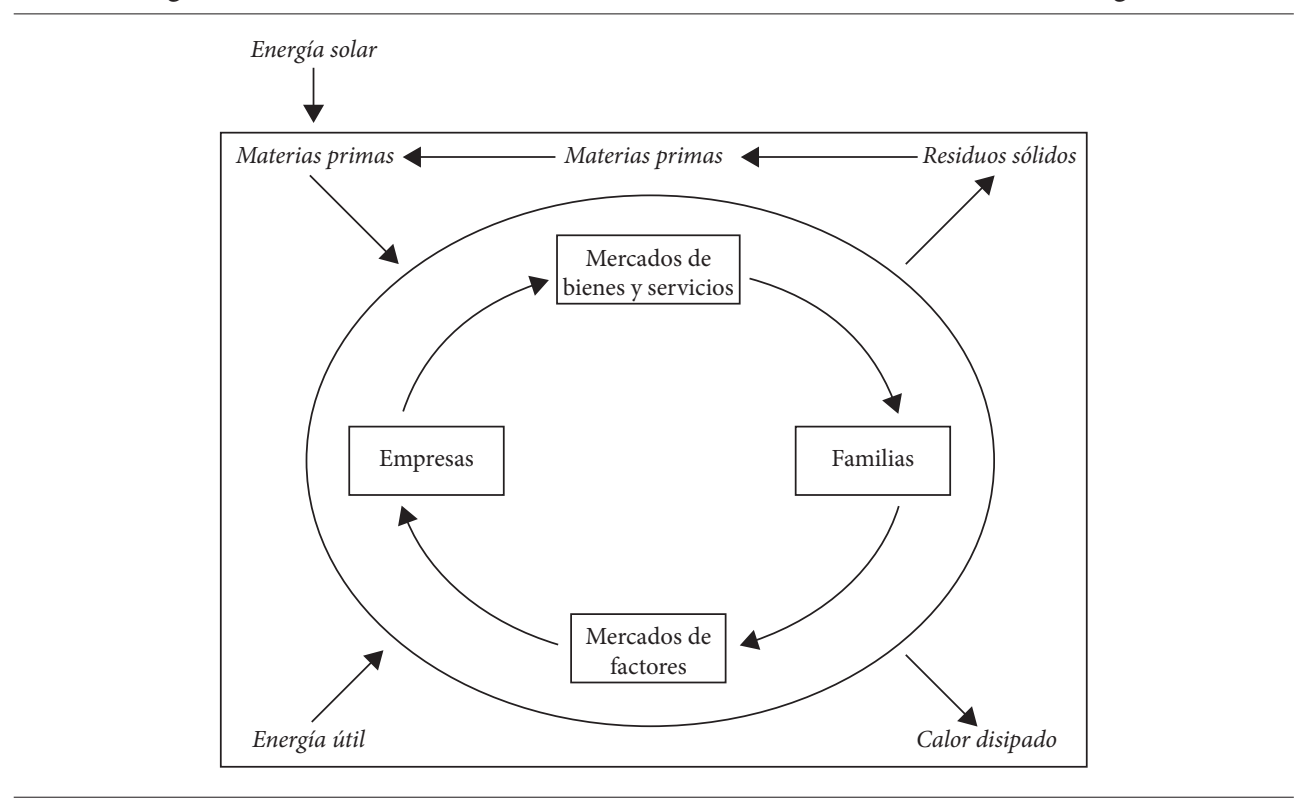

Fuente: adoptado de Martínez-Alier (2006).

(Van Alstine y Neumayer, 2010, p. 2). Los argumentos de esta tendencia son entre otros que: Con mayores ingresos el desarrollo tecnológico se aceleraría permitiendo por medio de éste reducir los impactos ambientales, se incrementaría la demanda de productos y servicios que tienen menor intensidad en material y crecería la demanda por un ambiente de calidad.

En lo que concierne a la última afirmación, existen teorías que mantienen que aquellas economías ricas caracterizadas por tener elevados niveles de ingresos, empiezan a valorar de distinta manera a la naturaleza, considerándola como un bien de lujo que requiere de mayor cuidado y protección. Sin embargo, esta afirmación implicaría que únicamente aquellos países ricos podrían adquirirlo, mientras que el resto de economías no podrían acceder a un ambiente limpio.

Por otro lado, los pesimistas, científicos de las ramas físicas y sociales, consideraban que la expansión de la economía requeriría una enorme y creciente cantidad de materiales y energía, lo cual generaría tal cantidad de desechos que la capacidad de asimilación de los sistemas ambientales sería sobrepasada. El resultado sería un colapso ambiental por la degradación generada y con ello la caída del bienestar humano.

Cabe mencionar que la tendencia pesimista no consideró a los avances tecnológicos para realizar sus análisis (Van Alstine y Neumayer, 2010, p. 2). Si bien la tecnología no es la solución para enfrentar la problemática ambiental en la magnitud que lo afirman los optimistas, tampoco podemos ignorar que sí se han generado tecnologías que permiten reducir en parte los impactos ambientales. 


\section{EL MITO DE LAS POSIBILIDADES ILIMITADAS}

Se sostuvo en secciones previas que la riqueza material y económica es necesaria para alcanzar el bienestar social. Sin embargo, esto no implica que en nombre del bienestar social se pueda considerar sobrepasar los límites que se encuentran claramente definidos.

Estos límites son establecidos en relación a dos factores fundamentales. El primero es la naturaleza finita de los recursos ecológicos que hacen posible la vida en el planeta. Estos recursos incluyen, obviamente, los de origen natural: combustibles fósiles, minerales, madera, agua, tierra, etc. Pero también abarcan la capacidad regenerativa de los ecosistemas, la diversidad de especies y la integridad de la atmosfera, de los suelos y de los océanos [...]. El segundo factor que limita nuestra capacidad para vivir bien es la magnitud de la población humana global. (Jackson, 2011, p. 72)

Si bien no se puede establecer cuál es el límite exacto para cada recurso, se ha tornado evidente que la actividad de los seres humanos se encuentra afectando y amenazando al adecuado funcionamiento de los ecosistemas. Esto, sumado al aumento sostenido de la población, genera un incremento acelerado de las presiones y daños ambientales.

No es posible sostener el mito de las posibilidades ilimitadas en un planeta con recursos finitos. Ignorar los límites evidentes únicamente implica «condenar a nuestros descendientes - y a las demás criaturas del mundo natural- a vivir en un planeta empobrecido» (Jackson, 2011, p. 72).

El ambiente cumple con varios roles indispensables para conservar la armonía de los ecosistemas y sustentar la vida. Los ecosistemas abastecen a los seres humanos de recursos naturales indispensables para la vida, tal como el aire y el agua; además es fuente de recursos para los procesos productivos dentro del sistema económico, como madera para construcción, recursos energéticos y minerales, etc. Otra de sus funciones esenciales es la asimilación de los residuos, como por ejemplo la absorción de carbono, la depuración de aguas contaminadas, el procesamiento natural de residuos sólidos, etc. Sobre esta función es importante mencionar que los ecosistemas tienen una limitada capacidad de resiliencia; una vez sobrepasada, la naturaleza no es capaz de procesar los residuos generándose daños que pueden llegar a ser irreversibles. Es decir que para mantener el equilibrio ambiental no se debería exceder esta capacidad de asimilación.

Una tercera función del ambiente es la de brindar diversos servicios como regulación del hábitat, provisión de elementos decorativos y recreativos, como sistema de prevención de la erosión y de inundaciones, etc. (Gómez-Baggethun y de Groot, 2007, p. 7).

\section{EL LIMITADO ALCANCE DEL PIB PARA MEDIR EL BIENESTAR}

El principal agregado económico calculado dentro de los sistemas de contabilidad nacional, y el indicador de mayor uso entre las economías de todo el mundo es el denominado Producto Interno Bruto (PIB), que mide el nivel de actividad económica de un país, reflejando el valor monetario del flujo de bienes y servicios en un período determinado. Desde sus inicios, los sistemas de contabilidad nacional, y su principal agregado el PIB, han recibido numerosas críticas. Partiendo de la broma de Paul Samuelson, premio Nobel de Economía en 1970, conocida desde los años cincuenta, con la que señalaba que si un profesor se casa con su sirvienta, 
el PIB sufriría a una disminución (Martínez-Alier y Roca, 2006). Sin embargo, con el pasar de los años se ha ido posicionando erróneamente como un indicador que refleja el desarrollo de las economías y el bienestar de sus habitantes.

Se volvió común que los ciudadanos de un país sientan que los costos de vida incrementaron rápidamente y en gran magnitud, reflejados en la caída de sus estándares de vida; mientras que las estadísticas presentadas por el gobierno presentan un panorama totalmente diferente con pequeños niveles de inflación, desempleo, etc.

La disparidad en las percepciones, refleja el uso inadecuado de los indicadores económicos, y también la falta de incorporación de las complejas relaciones del sistema económico y social.

Los sistemas estadísticos son herramientas para la gestión económica, pero sirven a una multiplicidad de objetivos. Nuestros sistemas estadísticos fueron creados originalmente para proporcionar una evaluación del desempeño de la economía de mercado y, en particular, para controlar las fluctuaciones cíclicas. Esto es todavía una función crítica. Pero las métricas desarrolladas para este propósito se utilizan a menudo (mal) como una medida de bienestar de la sociedad. Esto se justificaría sólo en un conjunto muy específico - y a menudo poco realistade supuestos. (Stiglitz et al., 2009, p. 7)

La contabilidad nacional entrega una impresión falsa del desempeño económico, considerando que se miden los bienes que son producidos, pero no llegan a considerar los males ocasionados (Stiglitz et al., 2009, p. 7). El PIB es un importante indicador económico, sin embargo, los análisis de los métodos de cálculo del PIB han reflejado que existen múltiples falencias.

En primer lugar, no se considera el autoconsumo, las externalidades y distorsiones del mercado, dentro de lo cual se incluyen las externalidades negativas generadas por el consumo de los recursos naturales y el daño causado al ambiente, es decir, el agotamiento y la degradación. Otros problemas también ampliamente analizados constituyen la desigualdad en la distribución del ingreso nacional, las actividades no remuneradas, el ocio, entre otros (Schuldt, 1995, p. 30).

La visión economicista del desarrollo, a través de indicadores agregados como el PIB, considera como positivos, sin discriminación, todos los procesos donde ocurren transacciones de mercado, sin importar si éstas son productivas, improductivas o destructivas. Resulta así, que la depredación indiscriminada de los recursos naturales hace aumentar el PIB, como lo hace una población enferma cuando incrementa su uso de drogas farmacéuticas o de servicios hospitalarios. (Max-Neef, 1986, p. 57; citado en Schuldt, 1995, p. 31)

\section{UNA PROPUESTA ALTERNATIVA: EL DECRECIMIENTO ECONÓMICO}

Una de las propuestas más recientes en la corriente de la economía ecológica es el decrecimiento económico. Sin embargo, el término surge mucho antes en el año 1979 en la introducción escrita por Jacques Grinevald e Ivo Rens para el libro de Georgescu-Roegen. El decrecimiento sostenible surge como una propuesta ante la realidad de la incompatibilidad entre crecimiento económico y sostenibilidad ecológica. Esta iniciativa se refiere al decrecimiento en términos de indicadores reales, es decir, que se debe generar una reducción de la energía y los materiales que ejercen presiones sobre el ambiente, como es el caso de los combustibles fósiles, los minerales, 
etc. También considera la reducción de aquellas causas de la reducción de la biodiversidad, es decir, el crecimiento de la población y el consumo o apropiación de la biomasa.

Para Martínez-Alier, el decrecimiento presenta algunas dificultades sociales. Una primera implica el aumento del desempleo ante el incremento de la productividad del trabajo en ausencia de crecimiento económico. La respuesta es doble. Los aumentos de productividad no están bien medidos. Por ejemplo, si hay una sustitución de energía humana por energía de máquinas, ¿los precios de esta energía tienen en cuenta el agotamiento de recursos, las externalidades negativas? Sabemos que no es así. Además, hay que separar más que actualmente el derecho a recibir una remuneración del hecho de que uno tenga empleo asalariado. Esa separación ya existe en muchos casos (niños y jóvenes, pensionistas, personas que perciben el seguro de desempleo), pero debe ampliarse más. Hay que redefinir el significado de «empleo» (teniendo en cuenta los servicios domésticos no remunerados, y todo el sector del voluntariado) y hay que introducir o ampliar la cobertura de la Renta de Ciudadano. (Martínez-Alier, 2008, p. 7-8).

Otro cuestionamiento es cómo se pagaría la deuda pública si la economía no crece. Sin embargo, esta pregunta no debe tener respuesta considerando que no se puede forzar a la economía a expandirse según la medida de los intereses monetarios aplicados a las deudas.

[...] También se objetará que si un país no crece económicamente, los capitales emigrarán a países donde las tasas de ganancia son mayores porque sus economías crecen. La respuesta es que el razonamiento es exacto, y que al fin y al cabo no es mala idea que el ahorro de un país rico que no quiere crecer más se traduzca (más allá de la reparación y amortización del capital físico propio) en inversiones y donaciones incorporadas en tecnologías que sean ambientalmente lo menos dañinas posible, hacia países pobres que deben crecer todavía, vigilando sin embargo cuál es la marcha de sus indicadores físicos que finalmente deben dejar de crecer. El movimiento del Decrecimiento Sostenible debe ser internacional. (Martínez-Alier, 2008, p. 8)

\section{BIBLIOGRAFÍA}

Daly, H. (1996). Beyond Growth. Boston, USA: Beacon Press.

Daly, H. (2005). Economics in a full world. Scientific American, (293). Recuperado de: http://sef.umd. edu/files/ScientificAmerican_Daly_05.pdf

Falconí, F. (2002). Economía y desarrollo sostenible: ¿Matrimonio feliz o divorcio anunciado? El caso de Ecuador. Quito, Ecuador: Flacso-Ecuador.

Gómez-Baggethun, E. y De Groot, R. (2007). Capital natural y funciones de los ecosistemas: explorando las bases ecológicas de la economía. Recuperado de: http://www.agro.uba.ar/users/semmarti/ Servicios/Servicios \%20de\%20los\%20Ec osistemas,\%20revista\%20ecosistemas.pdf

Jackson, T. (2011). Prosperidad sin crecimiento. Economía para un planeta finito. Barcelona, España: Icaria.

Kerschner, Ch. (2008). Economía en estado estacionario vs. decrecimiento económico: ¿opuestos o complementarios? Ecología política, (35). Recuperado de: http://ecologiapolitica.info/ep/35.pdf

Kerschner, Ch. (2009). La Economía del Estado Estacionario: ¿El único camino hacia un futuro sostenible?. Recuperado de: http://virtual.uptc.edu.co/revistas/index.php/cenes/article/viewFile/600/536.

Keynes, J. M. (1936). The General Theory of Employment, Interest and Money. USA: Macmillan Cambridge University Press. 
Martínez-Alier, J. (2008). Decrecimiento Sostenible. Instituto de Estudios Ecologistas del Tercer Mundo. Recuperado de: http://www. estudiosecologistas.org/docs /reflexión/Desdesarrollo/decrecimiento.pdf

Martínez-Alier, J. y Roca, R. (2006). Economía ecológica y política ambiental. México DF, México: Fondo de Cultura Económica.

Max-Neef, M. (1986). Economía descalza. Buenos Aires, Argentina: Nordan.

Meadows, D., Meadows, D., Randers, J. y Behrens, W. (1972). Los límites del crecimiento. México DF, México: Fondo de Cultura Económica.

Miller, R. (2002). Economía hoy. Bogotá, Colombia: Addison Wesley.

Munda, G. (1997). Environmental Economics, Ecological Economics, and the Concept of Sustainable Development. Recuperado de: http://www.jstor.org /stable/30301601

Schuldt, J. (1995). Repensando el desarrollo: Hacia una concepción alternativa para los países andinos. Quito, Ecuador: Centro Andino de Acción Popular.

Stiglitz, J., Sen, A. y Fitoussi, J-P. (2009). Report by the Commission on the Measurement of Economic Performance and Social Progress. Commission on the Measurement of Economic Performance and Social Progress. Recuperado de: http://www.stiglitz-sen-fitoussi.fr/en/index.htm

Van Alstine, J. y Neumayer, E. (2010). The Environmental Kuznets Curve. Geography and Environment - London School of Economics. Recuperado de: http://www2.lse.ac.uk/ geographyAndEnvironment/whosWho /profiles/neumayer/pdf/EKC.pdf 
